\title{
Article \\ Optimization of Line-Tunneling Type L-Shaped Tunnel Field-Effect-Transistor for Steep Subthreshold Slope
}

\author{
Faraz Najam 1 and Yun Seop Yu* *(D) \\ Department of Electrical, Electronic and Control Engineering and IITC, Hankyong National University, \\ Anseong 17579, Korea; faraznajam@hknu.ac.kr \\ * Correspondence: ysyu@hknu.ac.kr; Tel.: +81-31-670-5293
}

Received: 12 October 2018; Accepted: 22 October 2018; Published: 24 October 2018

\begin{abstract}
The L-shaped tunneling field-effect-transistor (LTFET) has been recently introduced to overcome the thermal subthreshold limit of conventional metal-oxide-semiconductor field-effect-transistors (MOSFET). In this work, the shortcomings of the LTFET was investigated. It was found that the corner effect present in the LTFET effectively degrades its subthreshold slope. To avoid the corner effect, a new type of device with dual material gates is presented. The new device, termed the dual-gate (DG) LTEFT (DG-LTFET), avoids the corner effect and results in a significantly improved subthreshold slope of less than $10 \mathrm{mV} / \mathrm{dec}$, and an improved ON/OFF current ratio over the LTFET. The DG-LTFET was evaluated for different device parameters and bench-marked against the LTFET. This work presents the optimum configuration of the DG-LTFET in terms of device dimensions and doping levels to determine the best subthreshold, ON current, and ambipolar performance.
\end{abstract}

Keywords: band-to-band tunneling; L-shaped tunnel field-effect-transistor; double-gate tunnel field-effect-transistor; corner-effect

\section{Introduction}

Tunnel field-effect-transistors (TFETs) are being actively pursued as a potential replacement to conventional metal-oxide-semiconductor (MOS) technology [1]. TFETs offer a sub-thermal subthreshold slope (SS) but suffer from limited ON current $I_{\mathrm{ON}}$ performance [2]. To overcome the limit, different types of line tunneling type TFETs have been introduced, including L-shaped [3] (LTFETs), U-shaped [4] (UTFETs), and Z-shaped [5] TFETs (ZTFETs). Among them, only the LTFET has been experimentally demonstrated [3].

It was found using device simulations that the 2D corner effect [6] present in LTFETs degrades its subthreshold performance. In order to remove SS degradation due to the kink effect induced by the source corner, the fully depleted rounded corner with a gradual doping profile was used [6]. The LTFET still achieves a sub-thermal SS, but as shown in this work there is room for significant improvement in the subthreshold performance of LTFETs. To achieve this improvement, a new device based on the original LTFET is introduced. The new device uses a dual-gate (DG) structure and is termed the DG-LTFET. The two gates (gate1 and gate2) have different workfunctions and different heights. The DG-LTFET was thoroughly evaluated for different device parameters, including the source region height, gate1 and gate2 heights, gate1 and gate 2 workfunctions, channel thickness, and drain doping levels. Optimum dimensions and drain doping level were determined for the DG-LTFET. Section 2 briefly discusses the corner-effect problem of the LTFET. Section 3 introduces the DG-LTFET and compares its results with the LTFET. Section 4 presents the conclusion. 


\section{The LTFET: The Corner Effect}

Figure 1 shows a schematic for LTFET. The $\mathrm{p}^{+}\left(10^{20} \mathrm{~cm}^{-3}\right)$ doped source region overlaps the gate with the $\mathrm{n}^{-}\left(10^{12} \mathrm{~cm}^{-3}\right)$ channel sandwiched in between them. This sandwiched channel region is termed as $R_{\text {nonoffset }}$. There is also a part of the channel termed $R_{\text {offset }}$ in which there is an offset present between the source and the gate, as indicted in Figure 1. The following parameters were used for all devices considered in this work unless otherwise specified: source height $\left(H_{\mathrm{S}}\right)=40 \mathrm{~nm}$, oxide thickness $\left(t_{\mathrm{ox}}\right)=2 \mathrm{~nm}$, length of $R_{\text {nonoffset }}\left(T_{\mathrm{j}}\right)=5 \mathrm{~nm}$, channel length $\left(L_{\mathrm{ch}}\right)=50 \mathrm{~nm}$, height of $R_{\text {offset }}\left(H_{\text {offset }}\right)=10 \mathrm{~nm}$, height of $R_{\text {nonoffset }}\left(H_{\text {nonoffset }}\right)=H_{\mathrm{s}}$, gate height $\left(H_{\mathrm{g} 1}\right)=H_{\mathrm{s}}+\left(H_{\mathrm{offset}}-t_{\mathrm{ox}}\right)=48 \mathrm{~nm}$, dielectric permittivity $\varepsilon_{\mathrm{ox}}=25$, metal gate workfunction $W_{\text {rk_LTFET }}=4.72 \mathrm{eV}$, and drain doping $\left(N_{\mathrm{d}}\right)=10^{20} \mathrm{~cm}^{-3}$.

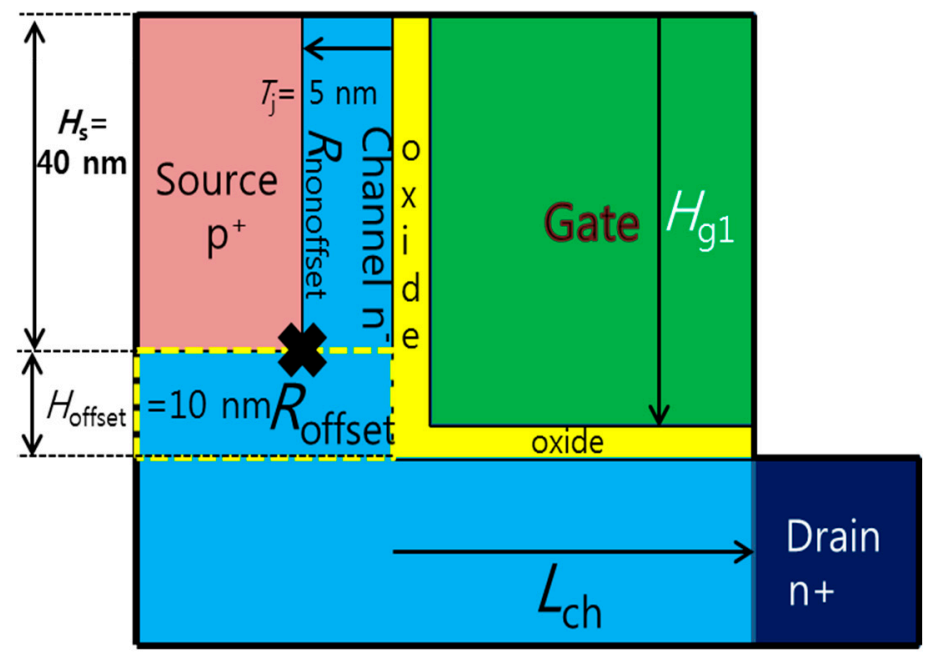

Figure 1. Schematic of the L-shaped tunneling field-effect-transistor (LTFET).

Sentaurus technology-computer-aided-design tool (TCAD) was used as the simulator [7]. The following models were used in the simulation: the dynamic nonlocal band-to-band-tunneling (BTBT) model, Fermi statistics, and the constant mobility model. The dynamic nonlocal BTBT model calculates BTBT in both lateral and 1D directions. Crystal orientation is assumed to be $<100>$ in all devices. A constant electron effective tunneling mass of $0.19 m_{\mathrm{o}}$ was used in all simulations [8]. All simulations were performed at a drain source bias $V_{\mathrm{ds}}=0.1 \mathrm{~V}$ unless otherwise specified.

For analysis to follow, drain-source current $\left(I_{\mathrm{ds}}\right)$ versus gate-source bias $\left(V_{\mathrm{gs}}\right)$ characteristics of the LTFET are shown in Figure 2a. There is a direct overlap between gate and source in $R_{\text {nonoffset, }}$ and the electric field in $R_{\text {nonoffset }}$ is in the $1 \mathrm{D}$ direction. In $R_{\text {offset }}$, however, the electric field from the gate converges around the sharp source corner marked by an $\mathbf{X}$ in Figure 1. This increases the potential in $R_{\text {offset }}$ as compared to $R_{\text {nonoffset }}$ for any given bias (until potential saturates due to electron inversion). Figure $2 \mathrm{~b}$ shows the surface potential at $V_{\mathrm{gs}}=0 \mathrm{~V}$. It can be seen that, because the electric field converges around the sharp source corner [6], the potential in $R_{\text {offset }}$ has increased. Since the potential is higher in $R_{\text {offset }}$ as compared to $R_{\text {nonoffset }}$, the threshold voltage for BTBT in $R_{\text {offset }}\left(V_{\text {th_Roffset }}\right)$ is lower than the threshold voltage for BTBT in $R_{\text {nonoffset }}\left(V_{\text {th_Rnonoffset }}\right)$.

Figure $3 \mathrm{a}, \mathrm{b}$ show the tunneling rate $\left(G_{\text {tun }}\right)$ contour plot and $G_{\text {tun }}$, respectively, at $V_{\mathrm{gs}}=0.21 \mathrm{~V}$ which is the bias needed to generate $I_{\mathrm{ds}}=10^{-13} \mathrm{~A}$ (from Figure 2a). It is obvious from Figure 3 that the BTBT only takes place in $R_{\text {offset }}$, whereas $R_{\text {nonoffset }}$ is completely switched off. Figure 4 a shows $G_{\text {tun }}$ at several $V_{\text {gs }}$ values. From Figure $4 \mathrm{a}, V_{\text {th_Roffset }}$ and $V_{\text {th_Rnonoffset }}$ can be found to be around $V_{\text {gs }}=0.17 \mathrm{~V}$ and $0.24 \mathrm{~V}$, respectively. Figure $4 \mathrm{~b}$ shows the $G_{\text {tun }}$ contour plot at $V_{\text {gs }}=V_{\text {th_Rnonoffset }}=0.24 \mathrm{~V}$. Figure $4 \mathrm{a}$ shows that $G_{\text {tun }}$ in $R_{\text {nonoffset }}$ just after it turns on, is always higher and has a much larger BTBT area (in the $y$ direction) as compared to $R_{\text {offset }}$. Thus, whenever $R_{\text {nonoffset }}$ turns on, it dominates over $R_{\text {offset }}$. The reason why $G_{\text {tun }}$ is higher in $R_{\text {nonoffset }}$ is simply because the BTBT paths in $R_{\text {offset }}$ are laterally 
oriented or $2 \mathrm{D}$ from source to the surface in $R_{\text {offset }}$, whereas the BTBT paths in $R_{\text {nonoffset }}$ are $1 \mathrm{D}$. The $2 \mathrm{D}$ BTBT paths being naturally longer than the 1D paths result in a lower $G_{\text {tun }}$ in $R_{\text {offset }}$.

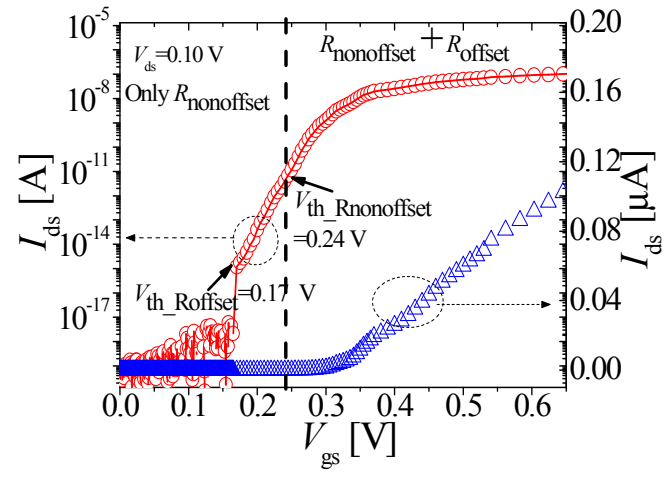

(a)

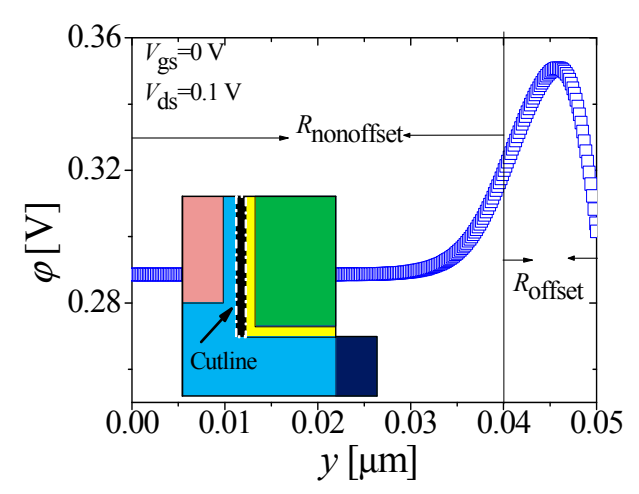

(b)

Figure 2. (a) $I_{\mathrm{ds}}-V_{\mathrm{gs}}$ transfer characteristics of the LTFET. $V_{\mathrm{th} \_ \text {Rnonoffset }}=0.24 \mathrm{~V}$ and $V_{\text {th } \_ \text {Roffset }}=0.17 \mathrm{~V}$. (b) Potential along the cutline shown in the inset at $V_{\mathrm{gs}}=0 \mathrm{~V}$. Potential is higher in $R_{\text {offset }}$.

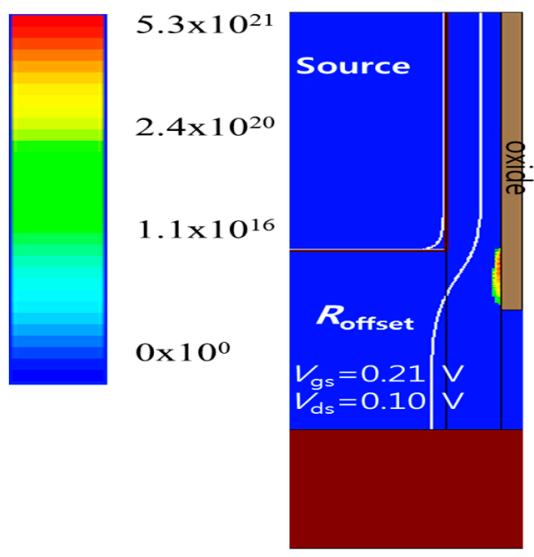

(a)

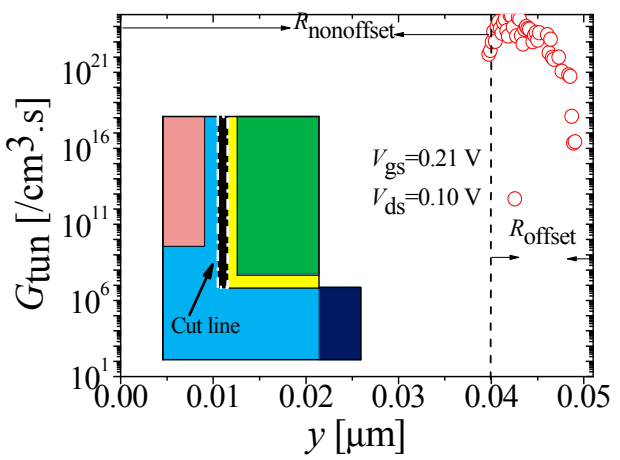

(b)

Figure 3. (a) $G_{\text {tun }}$ contour plot at $V_{\mathrm{gs}}=0.21 \mathrm{~V}$, which is the bias needed to generate $I_{\mathrm{ds}}=10^{-13} \mathrm{~A}$ and (b) G tun extracted from (a).

From Figure $4 \mathrm{a}$, it can be observed that, for a large part of the subthreshold region $\left(V_{\mathrm{gs}}<0.24 \mathrm{~V}\right)$, only $R_{\text {offset }}$ with the longer 2D BTBT paths and lower $G_{\text {tun }}$ is contributing to the BTBT current and the more efficient $R_{\text {nonoffset }}$ makes no contribution to the current. In other words, the LTFET underperforms in the subthreshold region. If $R_{\text {nonoffset }}$ could be forced to turn on at a lower bias than $R_{\text {offset}}$, which is the condition $V_{\text {th_Rnonoffset }}<V_{\text {th_Roffset, }} R_{\text {nonoffset }}$ will turn on in the subthreshold region, and with the condition $G_{\text {tun }}$ in $R_{\text {nonoffset }}>G_{\text {tun }}$ in $R_{\text {offset}}$, demonstrated in Figure 4a, a significant improvement in SS could be expected.

In other words, the $R_{\text {nonoffset }}$ could be regarded as a parasitic region with a parasitic, fringing capacitance originating from the bottom of the gate to the sharp source corner. Since the potential is different in this area (Figure 2b), the capacitance associated with this region is different from the $R_{\text {nonoffset }}$ region. If $V_{\text {th_Rnonoffset }}<V_{\text {th_Roffset }}$ could be achieved, as is demonstrated below, the effect of this parasitic capacitance could be practically eliminated, and this is the purpose of the device proposed below. Since drain is not in close proximity to $R_{\text {offset }} / R_{\text {nonoffset }}$, where the BTBT current is generated, gate-drain capacitance fringing capacitance is not expected to influence the potential and BTBT significantly at high frequency. 


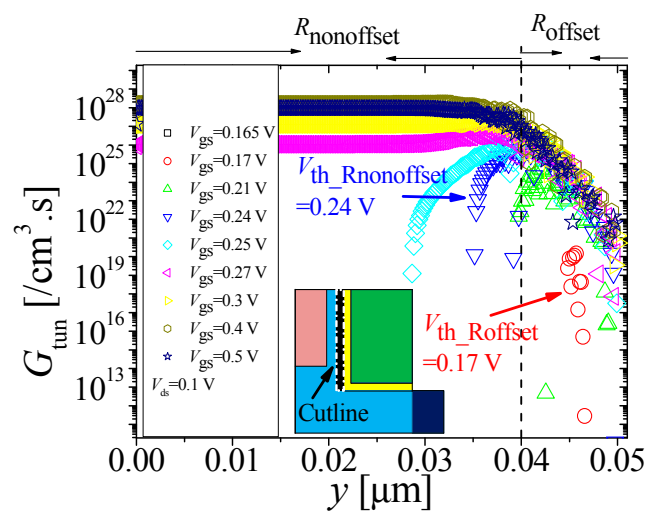

(a)

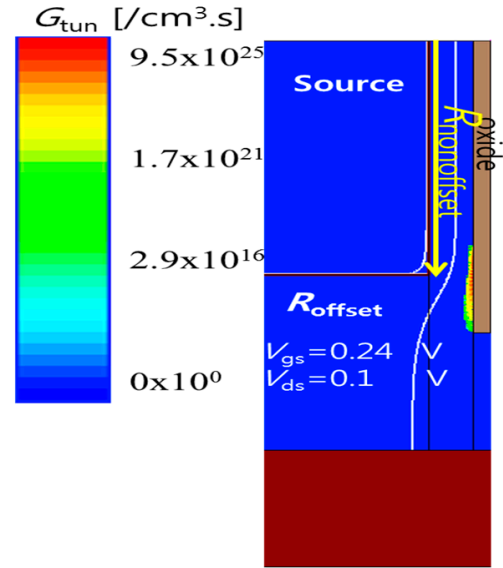

(b)

Figure 4. (a) $G_{\text {tun }}$ at different $V_{\text {gs }}$. (a) $V_{\text {th_Rnonoffset }}=0.24 \mathrm{~V}$ and $V_{\text {th_Roffset }}=0.17 \mathrm{~V}$. (b) $G_{\text {tun }}$ contour plot at $V_{\mathrm{gs}}=V_{\text {th_Rnonoverlap }}=0.24 \mathrm{~V}$. In (b), yellow arrow indicates the height of $R_{\text {nonoffset }}$.

\section{DG-LTFET}

\subsection{The DG-LTFET: Basic Device Physics}

In order to achieve the condition $V_{\text {th_Rnonoffset }}<V_{\text {th_Roffset }}$, the DG-LTFET is presented in Figure 5 a. DG-LTFET uses dual material gates denoted by gate1 and gate2, each with a different workfunction $\left(W_{\text {rk_gate1/2 }}\right)$ and height $\left(H_{\mathrm{g} 1 / 2}\right) . H_{\mathrm{g} 1}=H_{\text {nonoffset }}=H_{\mathrm{s}}=40 \mathrm{~nm}, H_{\text {offset }}=10 \mathrm{~nm}, H_{\mathrm{g} 2}=H_{\text {nonoffset }}-$ $H_{\mathrm{g} 1}+\left(H_{\text {offset }}-t_{\mathrm{ox}}\right)=8 \mathrm{~nm}$, and $T_{\mathrm{j}}=5 \mathrm{~nm}$. $W_{\text {rk_gate1 }}$ is always lower than $W_{\text {rk_gate2. }} W_{\text {rk_gate2 }}$ is fixed at $W_{\text {rk LITET }}=4.72 \mathrm{eV}$ for all DG-LTFET considered in this work. The DG-LTFET process-flow is indicated in Figure 5a. The process-flow is based on the LTFET process-flow [3]. The DG-LTFET process-flow follows the LTFET process-flow until the chemical vapor deposition (CVD) of gate2 (similar to the gate deposition in the LTFET). After this, two additional steps are required. The device is masked to protect the gate oxide and channel areas, and gate2 is selectively etched according to the desired height. Gate1 is then deposited in the recess created by gate2-etching by a low-temperature atomic layer deposition process. Similar dual-material gate structures have been extensively reported in the literature including [9-11].

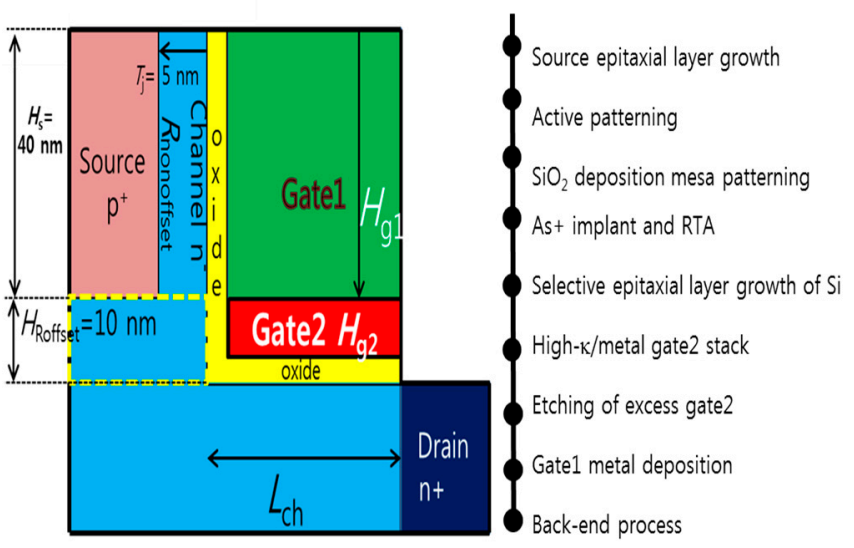

(a)

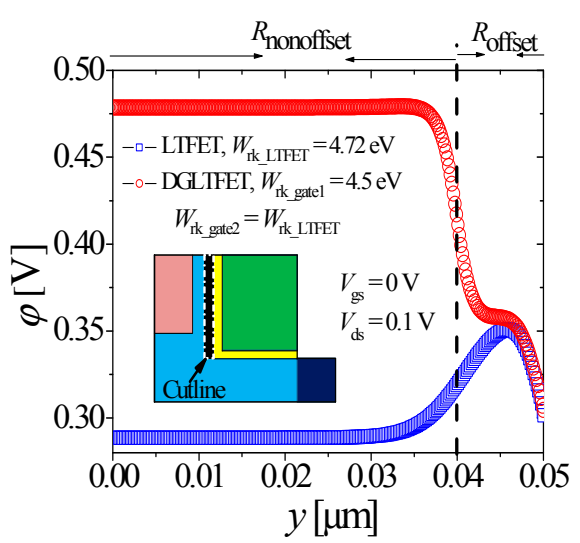

(b)

Figure 5. (a) Schematic of DG-LTFET with process-flow indicated alongside and (b) $V_{\mathrm{fb}}$ of DG-LTFET (red symbols) compared with that of the LTFET (blue symbols). In the DG-LTFET, $W_{\text {rk_gate } 1}=4.5 \mathrm{eV}$ and $W_{\text {rk_gate2 }}=W_{\text {rk_LTFET }}=4.72 \mathrm{eV}$ were used. 
Lower $W_{\text {rk_gate1 }}$ results in an increased flatband voltage [12] $\left(V_{\mathrm{fb}}\right)$ in $R_{\text {nonoffset }}$ as compared to $R_{\text {offset }}$. Figure $5 \mathrm{~b}$ shows $V_{\mathrm{fb}}$ of DG-LTFET (red symbols) with $W_{\text {rk_gate1 }}=4.5 \mathrm{eV}$ and $W_{\text {rk_gate2 }}=W_{\text {rk_LTFET }}$. Also shown for the reference is $V_{\mathrm{fb}}$ of the LTFET (blue symbols). Expectedly, the DG-LTFET potential increases in $R_{\text {nonoffset. }}$ The potential does not change abruptly from gate 1 to gate 2 because of the presence of $2 \mathrm{D}$ effects around the source corner. Electric field from the bottom of gate 2 converges around the source corner. Around the middle of $\mathrm{R}_{\text {offset, }}$ equilibrium is established between the two gates and DG-LTFET potential overlaps LTFET potential since $W_{\text {rk_gate2 }}=W_{\text {rk_LTFET }}$. With $W_{\text {rk_gate1 }}<W_{\text {rk_gate2, }}$ the increased potential in $R_{\text {nonoffset }}$ reduces $V_{\text {th_Rnonoffset }}$. If $W_{\text {rk_gate1/2 }}$ are appropriately tuned with $W_{\text {rk_gate1 }}<W_{\text {rk_gate2 }}$, the condition $V_{\text {th_Rnonoffset }}<V_{\text {th_Roffset }}=0.17 \mathrm{~V}$ can be achieved. Because $W_{\text {rk_gate2 }}$ $=W_{\text {rk_LTFET }}=4.72 \mathrm{eV}, V_{\text {th_Roffset }}$ (in the DG-LTFET) is equal to $V_{\text {th_Roffset }}$ (in the LTFET).

Figure 6a-c show $I_{\mathrm{ds}}-V_{\mathrm{gs}}$ characteristics at different $W_{\text {rk_gate1 }}, \mathrm{SS}$, and $I_{\mathrm{ON}} / I_{\mathrm{OFF}}$ of the DG-LTFET with constant $W_{\text {rk_gate2 }}=W_{\text {rk_LTFET }}=4.72 \mathrm{eV}$ for all DG-LTFET, respectively. Also shown for the reference is the $I_{\mathrm{ds}}-V_{\mathrm{gs}}$ characteristics of the LTFET (black squares). $I_{\mathrm{ON}}$ is extracted at $V_{\mathrm{gs}}=0.7 \mathrm{~V}$, and $I_{\mathrm{OFF}}$ is defined as $I_{\mathrm{ds}}=10^{-17}$ A. With $W_{\text {rk_gate1 }}=4.675 \mathrm{eV}$ (red circles), the $V_{\text {th_Rnonoffset }}$ is reduced to $0.189 \mathrm{~V}$. Compared with the LTFET, $R_{\text {nonoffset }}$ now turns on earlier in the subthreshold region, along with $R_{\text {offset }}$. Since the BTBT is more efficient in $R_{\text {nonoffset }}$ (Figure $4 \mathrm{a}$ ) as compared to $R_{\text {offset }}, I_{\mathrm{ds}}$ increases more rapidly within the subthreshold region.

Hence, just at the transition point, where $R_{\text {nonoffset }}$ turns on $\left(V_{\text {gs }} \sim 0.189\right)$, a kink appears in the $I_{\mathrm{ds}}-V_{\text {gs }}$ curve. With $W_{\text {rk_gate1 }}=4.65 \mathrm{eV}$ (green triangles), $V_{\text {th_Rnonoffset }}$ is reduced to $V_{\text {gs }}=0.167 \mathrm{~V}$ and the condition $V_{\text {th_Rnonoffset }}<V_{\text {th_Roffset }}$ is achieved, and DG-LTFET exhibits a remarkable SS with values less than $10 \mathrm{mV} / \mathrm{dec}$ as seen in Figure $6 \mathrm{~b}$. With $W_{\text {rk_gate1 }}=4.625 \mathrm{eV}$ (blue stars), $V_{\text {th_Rnonoffset }}$ reduces further to $0.1448 \mathrm{~V}$, which is $<V_{\text {th_Roffset }}$. If $V_{\text {th_Rnonoffset }}<V_{\text {th_Roffset }}$ is established, then any increase in $V_{\text {th_Roffset }}-V_{\text {th_Rnonoffset }}$ simply shifts the $I_{\mathrm{ds}}-V_{\text {gs }}$ to the left without any change in SS as shown by the blue stars $\left(W_{\text {rk_gate } 1}=4.625 \mathrm{eV}\right)$ and orange diamonds $\left(W_{\text {rk_gate } 1}=4.5 \mathrm{eV}\right)$ in Figure $6 \mathrm{a}, \mathrm{b}$, respectively. An improvement of $\sim 16 \%$ is observed in the $I_{\mathrm{ON}} / I_{\mathrm{OFF}}$ of the DG-LTFET (with $W_{\text {rk_gate } 1}=4.625 \mathrm{eV}$ ) over the LTFET.

Figure 7 a shows the $G_{\text {tun }}$ contour plot of DG-LTFET at a $V_{\text {gs }}(=0.172 \mathrm{~V})$ bias needed to achieve an equivalent $I_{\mathrm{ds}}$ of $10^{-13} \mathrm{~A}$ in DG-LTFET with $W_{\text {rk_gate } 1}=4.65 \mathrm{eV}$. Figure $7 \mathrm{~b}$ shows the contour plot extracted from Figure $7 \mathrm{a}$. For reference, Figure $7 \mathrm{~b}$ also shows that $G_{\text {tun }}$ needed to generate an equivalent amount of $I_{\mathrm{ds}}$ in the LTFET (at a $V_{\mathrm{gs}}$ bias of $0.21 \mathrm{~V}$, Figure $3 \mathrm{~b}$ ). As can be seen in Figure 7b, the LTFET needs contribution only from $R_{\text {offset }}$, but generating the same amount of $I_{\mathrm{ds}}$ DG-LTFET depends heavily on $R_{\text {nonoffset }}$ with some contribution from $R_{\text {offset }}$. Because $G_{\text {tun }}$ in $R_{\text {nonoffset }}$ is more efficient (Figure 4a), as the $V_{\text {gs }}$ bias increases, $G_{\text {tun }}$ increases exponentially in a much larger area in $R_{\text {nonoffset }}$, which results in the DG-LTFET exhibiting a much steeper subthreshold swing, while the LTFET continues to depend only on the inefficient BTBT in $R_{\text {offset }}$ until around $V_{\text {th_Rnonoffset }}=0.24 \mathrm{~V}$. 


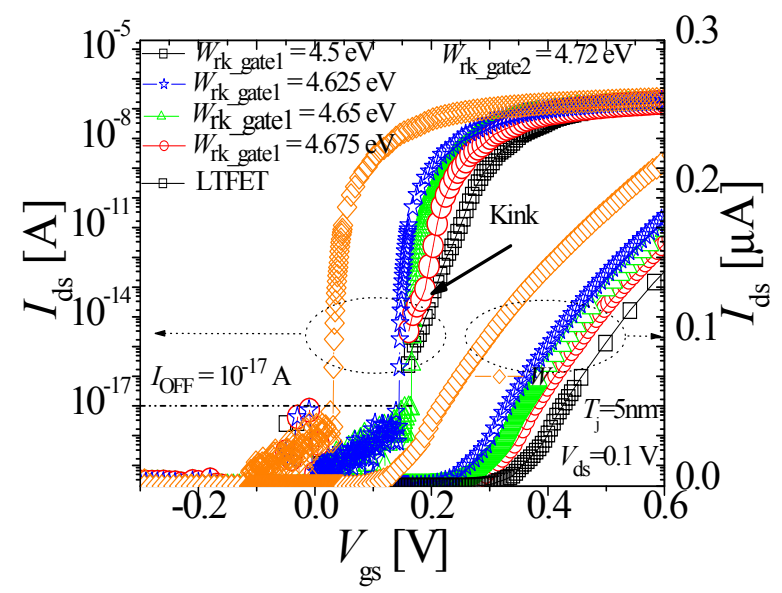

(a)

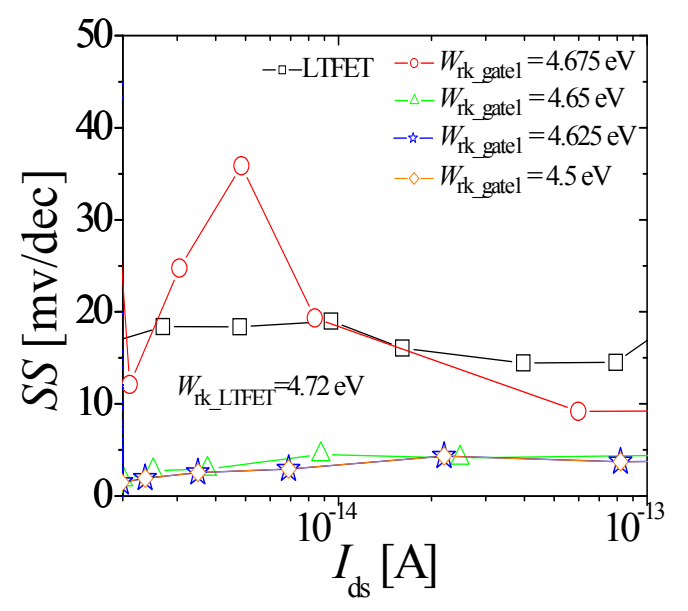

(b)

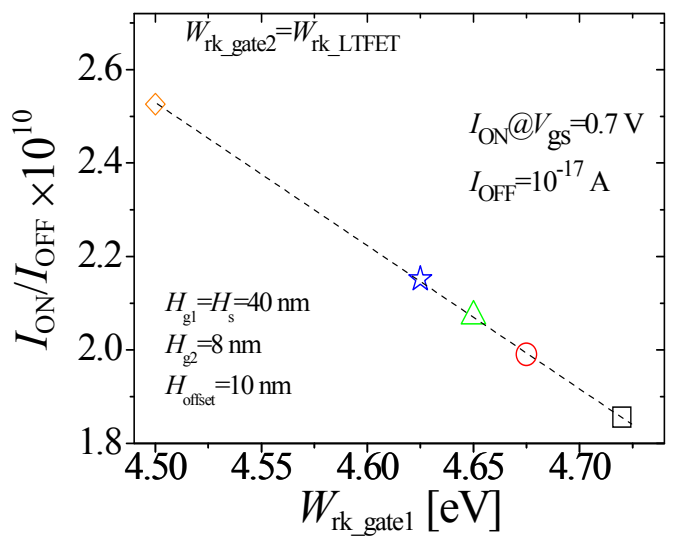

(c)

Figure 6. (a) $I_{\mathrm{ds}}-V_{\text {gs }}$ characteristics of DG-LTFET with different $W_{\text {rk_gate1 }} \mathrm{s}$ and fixed $W_{\text {rk_gate2 }}=W_{\text {rk_LTFET }}$. Also shown are $I_{\mathrm{ds}}-V_{\mathrm{gs}}$ characteristics of the LTFET (black squares). (b) SS extracted from $I_{\mathrm{ds}}-V_{\mathrm{gs}}$ characteristics in Figure 8a. (c) $I_{\mathrm{ON}} / I_{\mathrm{OFF}}$ ratio extracted from $I_{\mathrm{ds}}-V_{\mathrm{gs}}$ characteristics in Figure 8a. Red circles: $W_{\text {rk_gate } 1}=4.675 \mathrm{eV}$; green triangles: $W_{\text {rk_gate1 }}=4.65 \mathrm{eV}$; blue stars: $W_{\text {rk_gate1 }}=4.625 \mathrm{eV}$; orange diamonds: $W_{\text {rk_gate1 }}=4.5 \mathrm{eV}$. 


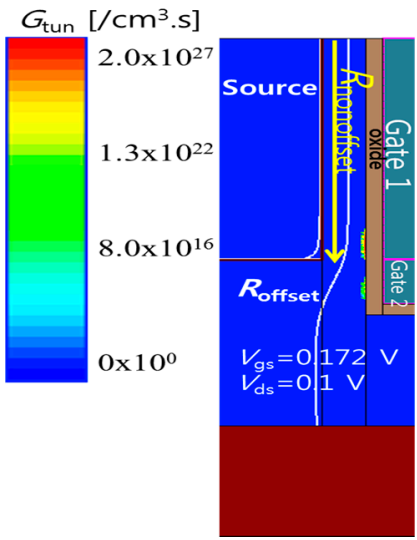

(a)

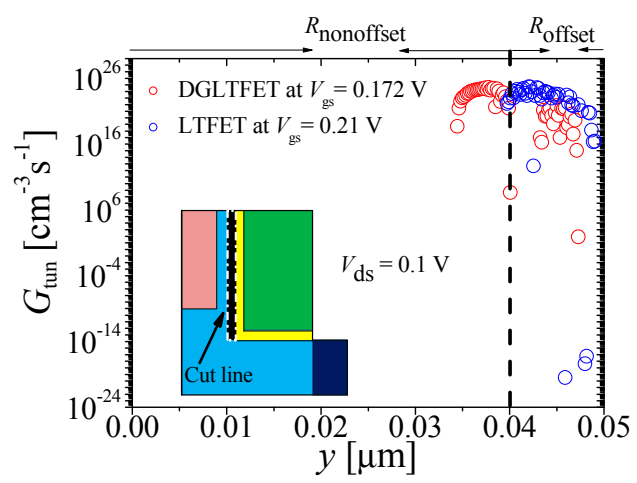

(b)

Figure 7. (a) $G_{\text {tun }}$ contour plot of DG-LTFET at $V_{\mathrm{gs}}=0.172 \mathrm{~V}$, which is needed to generate $I_{\mathrm{ds}}=10^{-13} \mathrm{~A}$ and (b) $G_{\text {tun }}$ extracted from (a) (red symbols). Also shown for reference is $G_{\text {tun }}$ (blue symbols) of the LTFET at a $V_{\mathrm{gs}}$ bias needed to generate $I_{\mathrm{ds}}=10^{-13} \mathrm{~A}$. In (a), yellow arrow indicates the height of $R_{\text {nonoffset }}$

\subsection{Device Optimization}

To optimize device performance, the impact of variations in key parameters including $H_{\mathrm{g} 1 / 2}$, $H_{\mathrm{s}} / T_{\mathrm{j}}$, and $N_{\mathrm{d}}$ was investigated. To investigate the impact of $H_{\mathrm{g} 1 / 2}$ values, $I_{\mathrm{ds}}-V_{\mathrm{gs}}$ characteristics for the DG-LTFET at different $H_{\mathrm{g} 1}$ and $H_{\mathrm{g} 2}=H_{\text {nonoffset }}-H_{\mathrm{g} 1}+\left(H_{\mathrm{offset}}-t_{\mathrm{ox}}\right)$ with fixed $W_{\text {rk_gate1 }}=4.5 \mathrm{eV}$ and $W_{\text {rk_gate2 }}=W_{\text {rk_LTFET }}, H_{\mathrm{s}}=H_{\text {nonoffset }}=40 \mathrm{~nm}, H_{\text {offset }}=10 \mathrm{~nm}$, and $T_{\mathrm{j}}=5 \mathrm{~nm}$ is presented in Figure 8. It can be seen that $I_{\mathrm{ds}}$ is independent of $H_{\mathrm{g} 1 / 2}$.

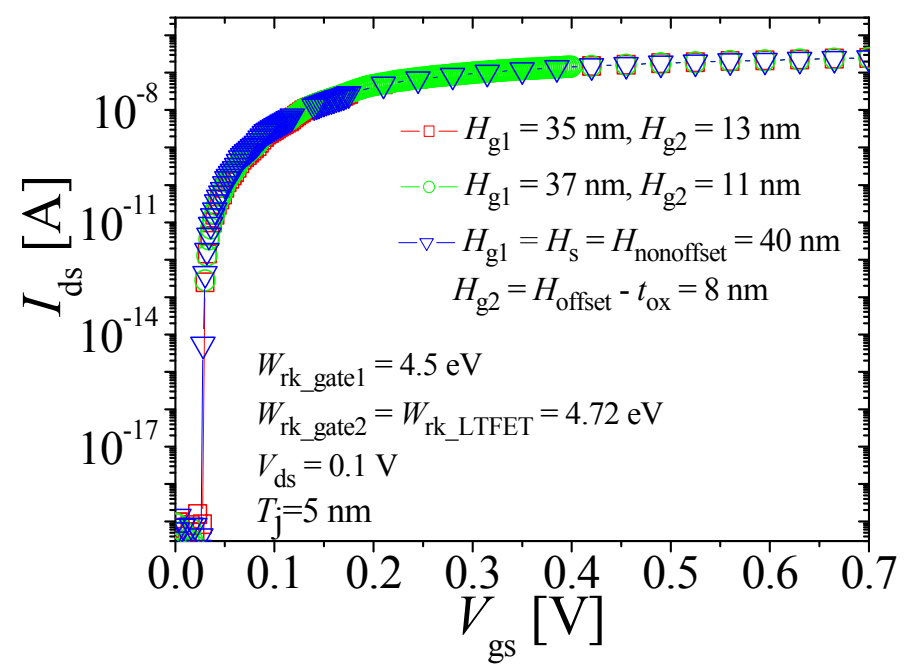

Figure 8. $I_{\mathrm{ds}}-V_{\mathrm{gs}}$ characteristics for several $H_{\mathrm{g} 1 / 2} \mathrm{~s}$ with $W_{\text {rk_gate1/2 }}=4.5 \mathrm{eV}$ and $W_{\text {rk_gate2 }}=W_{\text {rk_LTFET }}$. Red squares, green circles, and blue triangles: $H_{\mathrm{g} 1}=35,37$, and $40 \mathrm{~nm}$, respectively.

Next, to investigate the effect of $T_{\mathrm{j}}$ on device performance, $I_{\mathrm{ds}}-V_{\mathrm{gs}}$ characteristics, $\mathrm{SS}$, and $I_{\mathrm{ON}} / I_{\mathrm{OFF}}$ of DG-LTFET are presented for different $T_{\mathrm{j}}$ with fixed $W_{\text {rk_gate1 }}=4.5 \mathrm{eV}$ and $W_{\text {rk_gate2 }}=W_{\text {rk_LTFET }}$, $H_{\mathrm{g} 1}=H_{\text {nonoffset }}=40 \mathrm{~nm}, H_{\text {offset }}=10 \mathrm{~nm}$, and $H_{\mathrm{g} 2}=H_{\text {nonoffset }}-H_{\mathrm{g} 1}+\left(H_{\text {offset }}-t_{\mathrm{ox}}\right)=8 \mathrm{~nm}$ in Figure $9 \mathrm{a}-\mathrm{c}$, respectively. It was found that the increasing $T_{\mathrm{j}}$ results in a degradation of the $I_{\mathrm{ON}} / I_{\mathrm{OFF}}$ ratio. It is simply because of the increase in BTBT path length with the increase in $T_{\mathrm{j}}$. The $T_{\mathrm{j}}$ of $5 \mathrm{~nm}$ was found to be optimum in this work as any further reduction will bring significant quantum confinement effect into play, which is well known to degrade device performance [4,5,13-15]. 


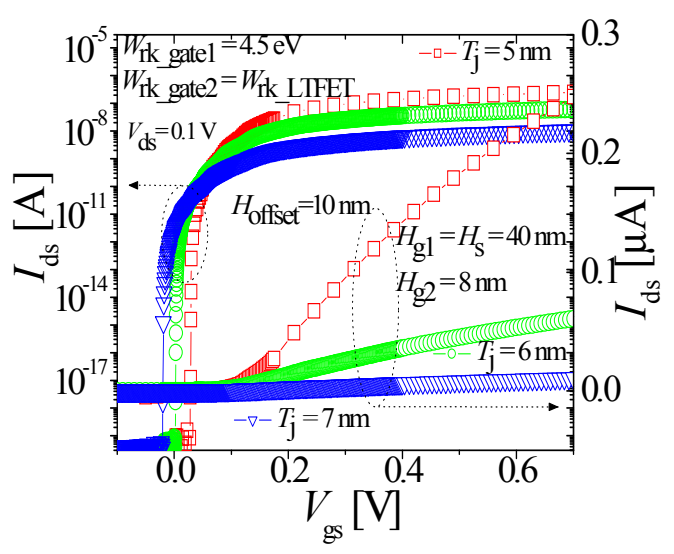

(a)

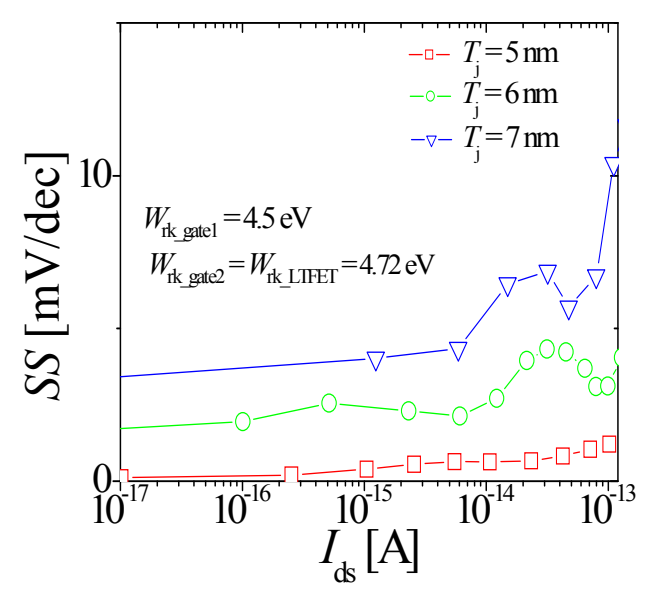

(b)

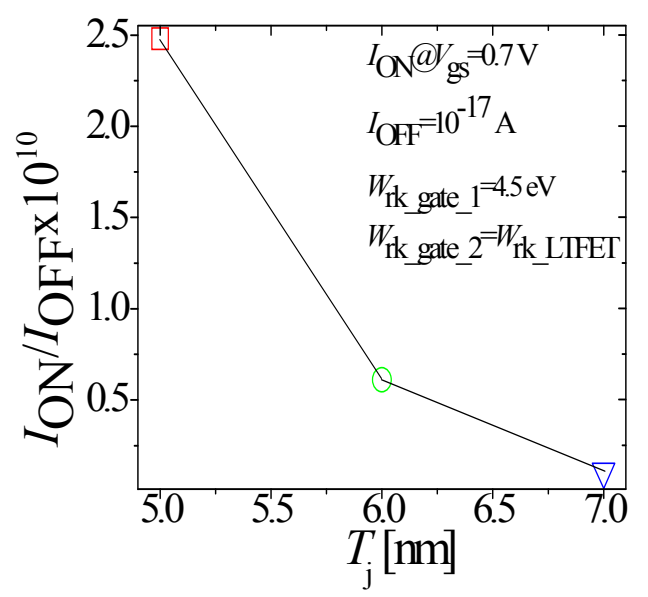

(c)

Figure 9. (a) $I_{\mathrm{ds}}-V_{\mathrm{gs}}$ characteristics of the DG-LTFET with different $T_{\mathrm{j}}$ and fixed $W_{\text {rk_gate1 }}=4.5 \mathrm{eV}$, $W_{\text {rk_gate2 }}=W_{\text {rk_LTFET }}$, and $H_{\mathrm{g} 1}=H_{\mathrm{s}}=H_{\text {nonoffset }}=40 \mathrm{~nm}, H_{\mathrm{g} 2}=H_{\text {offset }}(10 \mathrm{~nm})-t_{\mathrm{ox}}=8 \mathrm{~nm}$. (b) The SS of $I_{\mathrm{ds}}-V_{\mathrm{gs}}$ shown in Figure 8a. (c) $I_{\mathrm{ON}} / I_{\mathrm{OFF}}$ ratio of $I_{\mathrm{ds}}-V_{\mathrm{gs}}$ characteristics shown in Figure $8 \mathrm{a}$. Red squares, green circles, and blue triangles: $T_{\mathrm{j}}=5,6$ and $7 \mathrm{~nm}$, respectively. 
Next, the impact of varying $H_{\mathrm{s}}$ was investigated. $I_{\mathrm{ds}}-V_{\mathrm{gs}}$ characteristics of the DG-LTFET for several $H_{\mathrm{S}}$ with fixed $W_{\text {rk_gate1 }}=4.5 \mathrm{eV}$ and $W_{\text {rk_gate2 }}=W_{\text {rk_LTFET }}, H_{\mathrm{g} 1}=H_{\mathrm{S}}=H_{\text {nonoffset }}$, $H_{\mathrm{g} 2}=H_{\text {nonoffset }}-H_{\mathrm{g} 1}+\left(H_{\text {offset }}-t_{\mathrm{ox}}\right)=8 \mathrm{~nm}$, and $T_{\mathrm{j}}=5 \mathrm{~nm}$ is presented in Figure 10. By maintaining $H_{\mathrm{g} 1}=H_{\mathrm{s}}, H_{\text {offset }}=10 \mathrm{~nm}$, and $H_{\mathrm{g} 2}=8 \mathrm{~nm}$, the electric field vector distribution within the DG-LTFET remains the same as $\mathrm{H}_{\mathrm{s}}$ is varied, and the BTBT area simply scales with $H_{\mathrm{s}}$. An increase (decrease) in the BTBT area with $H_{\mathrm{S}}$ simply results in an increased (decreased) $I_{\mathrm{ON}} / I_{\mathrm{OFF}}$ ratio as shown in Figure 10b with no change in SS, as evident from Figure 10a.

Finally, the ambipolar current of the DG-LTFET is discussed. Ambipolar $I_{\mathrm{ds}}$ of TFET depends on the drain-channel junction. In the DG-LTFET, the drain-channel junction is controlled by gate2 with $W_{\text {rk_gate2 }}=W_{\text {rk_LTFET }}$. With the same workfunction, the electrostatics of the drain-channel junction in the DG-LTFET is exactly the same as that in the LTFET. Figure 11a shows ambipolar $I_{\mathrm{ds}}$ of the DG-LTFET compared with the LTFET. Any change in $W_{\text {rk_gate1 }}$ in the DG-LTFET does not affect the drain-channel junction. The same argument applies for any other design parameter variation in DG-LTFET including $H_{\mathrm{s}}, H_{\mathrm{g} 1 / 2}$, and $T_{\mathrm{j}}$; that is, as long as the electrostatics of the drain-channel junction remains unaffected, the DG-LTFET will exhibit an equivalent ambipolar $I_{\mathrm{ds}}$ as the LTFET. Further, the impact of $N_{\mathrm{d}}$ on ambipolar $I_{\mathrm{ds}}$ was considered. Different $N_{\mathrm{d}}$ values were considered for a DG-LTFET with $W_{\text {rk_gate1 }}=4.5 \mathrm{eV}$ and $W_{\text {rk_gate2 }}=W_{\text {rk_LTFET }}, H_{\mathrm{g} 1}=H_{\text {nonoffset }}=40 \mathrm{~nm}, H_{\mathrm{g} 2}=H_{\text {offset }}-t_{\text {ox }}=8 \mathrm{~nm}$, and $T_{\mathrm{j}}=5 \mathrm{~nm}$, and the results are shown in Figure 11b. A drain doping level of $10^{18} \mathrm{~cm}^{-3}$ was found to suppress ambipolar $I_{\mathrm{ds}}$ appreciably without affecting the $I_{\mathrm{ON}}$.

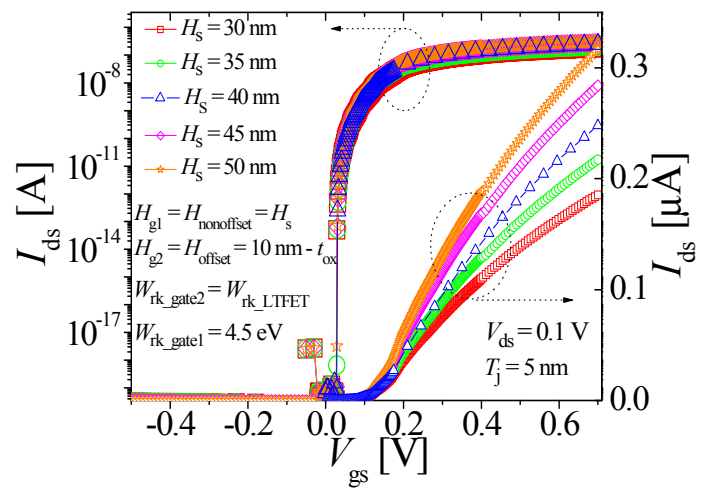

(a)

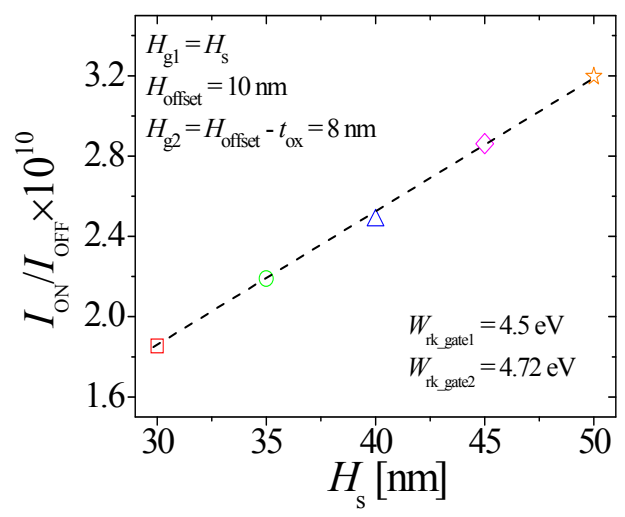

(b)

Figure 10. $I_{\mathrm{ds}}-V_{\text {gs }}$ characteristics of DG-LTFET with different $H_{\mathrm{s}}$, fixed $W_{\text {rk_gate1 }}=4.5 \mathrm{eV}, W_{\text {rk_gate2 }}$ $=W_{\text {rk_LTFET }}$, and $H_{\mathrm{g} 1}=H_{\mathrm{s}}=H_{\text {nonoffset }}, H_{\mathrm{g} 2}=H_{\text {offset }}(=10 \mathrm{~nm})-t_{\mathrm{ox}}=8 \mathrm{~nm}$. (b) An $I_{\mathrm{ON}} / I_{\mathrm{OFF}}$ ratio of $I_{\mathrm{ds}}-V_{\mathrm{gs}}$ characteristics shown in (a). Red squares, green circles, blue triangles, magenta diamonds, and orange stars: $H_{\mathrm{S}}=30,35,40,45$, and $50 \mathrm{~nm}$, respectively. 


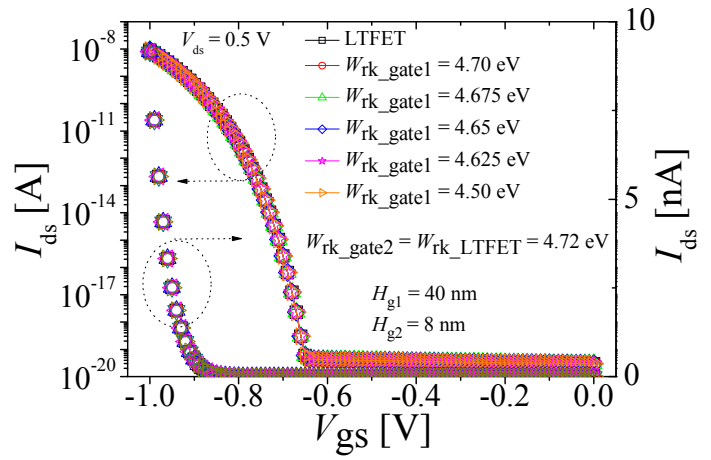

(a)

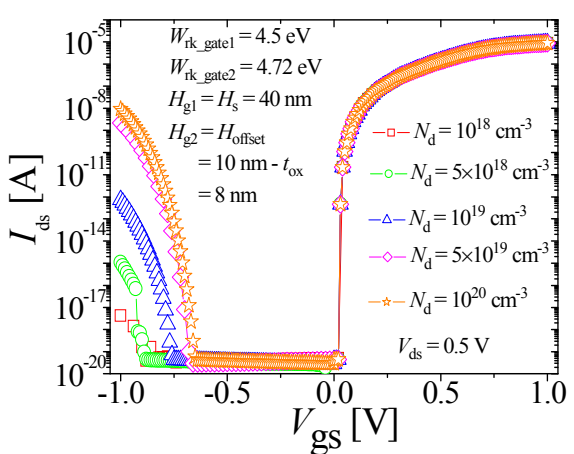

(b)

Figure 11. (a) $I_{\mathrm{ds}}-V_{\mathrm{gs}}$ characteristics of DG-LTFET at $V_{\mathrm{ds}}=0.5 \mathrm{~V}$ with different $W_{\text {rk gate } 1}$ and $W_{\text {rk_gate2 }}=W_{\text {rk_LTFET }}, H_{\mathrm{g} 1}=H_{\text {offset }}=10 \mathrm{~nm}, H_{\mathrm{g} 2}=8 \mathrm{~nm}, T_{\mathrm{j}}=5 \mathrm{~nm}$ and $N_{\mathrm{d}}=10^{20} \mathrm{~cm}^{-3}$. Red circles, green triangles, blue diamonds, magenta stars, and orange right triangles: $W_{\text {rk_gate1 }}=4.7$, 4.675, 4.65, 4.625, and $4.5 \mathrm{eV}$. (b) DG-LTFET $I_{\mathrm{ds}}$ with different $N_{\mathrm{d}} \cdot N_{\mathrm{d}}=10^{18} \mathrm{~cm}^{-3}$ demonstrates almost negligible ambipolar $I_{\mathrm{ds}}$. Red squares, green circles, blue triangles, magenta diamonds, and orange stars: $N_{\mathrm{d}}=10^{18}, 5 \times 10^{18}, 10^{19}, 5 \times 10^{19}$, and $10^{20} \mathrm{~cm}^{-3}$.

\section{Conclusions}

The device physics of the LTFET was investigated. It was found that a large part of the subthreshold region is dominated by the parasitic, lateral, 2D BTBT from the source to $R_{\text {offset }}$ with a lower $G_{\text {tun }}$. The more efficient 1D BTBT from the source to $R_{\text {nonoffset, }}$ with a higher $G_{\text {tun }}$ takes place at a higher bias in the subthreshold region. In other words, the condition, that is, $V_{\text {th_Rnonoffset }}>V_{\text {th_Roffset, }}$ exists in the LTFET. With $R_{\text {nonoffset }}$ not conducting the device does not utilize its channel fully during the subthreshold region. A new type of device based on the LTFET was introduced in this work. The device uses a dual gate structure with $W_{\text {rk_gate1 }}<W_{\text {rk_gate2 }}$. This increases the potential in $R_{\text {nonoffset }}$ and lowers $V_{\text {th_Rnonoffset }}$. The DG-LTFET reverses the threshold condition of the LTFET, that is, it lowers $V_{\text {th_Rnonoffset }}$ and makes it $<V_{\text {th_Roffset }} . R_{\text {nonoffset }}$ with higher $G_{\text {tun }}$ turns on earlier than $R_{\text {offset }}$ in the subthreshold region in the DG-LTFET and the device exhibits an SS of less than $10 \mathrm{mV} / \mathrm{dec}$. It was found that $W_{\text {rk_gate }}$ in the DG-LTFET needs to be sufficiently less than $W_{\text {rk_gate2 }}$ to achieve the sub $10 \mathrm{mv} / \mathrm{dec} \mathrm{SS}$. It was found that $I_{\mathrm{ds}}$ and SS are independent of $H_{\mathrm{g} 1 / 2}$. The DG-LTFET was further evaluated for different device dimensions including $T_{\mathrm{j}}$ and $H_{\mathrm{s}}$ while maintaining the electric field vector distribution equivalent. $I_{\mathrm{ds}}$ decreases with an increase in $T_{\mathrm{j}}$ and scales with $H_{\mathrm{s}}$. The $N_{\mathrm{d}}$ value of $10^{18} \mathrm{~cm}^{-3}$ was found to appreciably reduce ambipolar $I_{\mathrm{ds}}$. With the results presented in this work, the DG-LTFET could be considered as a viable potential replacement to conventional MOSFET and 3D integrations [16].

Author Contributions: Conceptualization, F.N. and Y.S.Y.; methodology, F.N. and Y.S.Y.; investigation, F.N. and Y.S.Y.; data curation, F.N.; writing-original draft preparation, F.N.; writing-review and editing, F.N., and Y.S.Y.; supervision, Y.S.Y.; project administration, Y.S.Y.; funding acquisition, Y.S.Y.

Funding: This research was funded by Ministry of Trade, Industry \& Energy (MOTIE), project number 10054888 and Korea Semiconductor Research Consortium (KSRC) support program for the development of future semiconductor devices.

Acknowledgments: This work was supported by IDEC (EDA tool).

Conflicts of Interest: The authors declare no conflict of interest. The funders had no role in the design of the study; in the collection, analyses, or interpretation of data; in the writing of the manuscript; or in the decision to publish the results. 


\section{References}

1. Avci, U.E.; Morris, D.H. Tunnel field-effect transistors. IEEE J. Electron. Devices Soc. 2015, 3, 88-95. [CrossRef]

2. Ionescu, A.M.; Riel, H. Tunnel field-effect transistors as energy efficient electronic switches. Nature 2011, 479, 329-337. [CrossRef] [PubMed]

3. Kim, S.W.; Kim, J.H.; Liu, T.K.; Choi, W.Y.; Park, B. Demonstration of L-shaped tunnel field-effect transistors. IEEE Trans. Electron. Devices 2016, 63, 1774-1778. [CrossRef]

4. Yang, Z. Tunnel field-effect transistor with an L-shaped gate. IEEE Electron. Device Lett. 2016, 4, 839-842. [CrossRef]

5. Imenabadi, R.M.; Saremi, M.; Vandenberghe, W.G. A novel PNPN-like Z-shaped tunnel field-effect transistor with improved ambipolar behavior and RF performance. IEEE. Trans. Electron. Devices 2017, 64, 4752-4758. [CrossRef]

6. Kim, S.W.; Choi, W.Y.; Sun, M.C.; Park, B.G. Investigation on the corner effect of L-shaped tunneling field-effect transistors and their fabrication method. J. Nanosci. Nanotechnol. 2016, 9, 6376-6381. [CrossRef]

7. Sentaurus User Manual; Version L-2016.03 March; Synopsys, Inc.: Mountain View, CA, USA, 2016.

8. Kao, K.H.; Verhulst, A.S.; Vandenberghe, W.G.; Soree, B.; Groeseneken, G.; Meyer, K.D. Direct and indirect band-to-band-tunneling in germanium-based TFETs. IEEE Trans. Electron. Devices 2012, 59, $292-301$. [CrossRef]

9. Saxena, R.S.; Kumar, M.J. Dual-material gate technique for enhanced transconductance and breakdown voltage of trench power MOSFETs. IEEE. Trans. Electron. Devices 2009, 56, 517-522. [CrossRef]

10. Long, W.; Ou, H.; Kuo, J.-M.; Chin, K.K. Dual-material gate (DMG) Field Effect Transistor. IEEE. Trans. Electron. Devices 1999, 46, 865-870. [CrossRef]

11. Polishchuk, I.; Ranade, P.; King, T.-J.; Hu, C. Dual work function metal gate CMOS technology using metal interdiffusion. IEEE Electron. Device Lett. 2001, 9, 444-446. [CrossRef]

12. Sze, S.M.; Kwok, K.N. Physics of Semiconductor Devices, 3rd ed.; John Wiley \& Sons: Hoboken, NJ, USA, 2006; ISBN 9780471143239.

13. Walke, A.M.; Verhulst, A.S.; Vandooren, A.; Verreck, D.; Simeon, E.; Rao, V.R.; Groeseneken, G.; Collaert, N.; Thean, A.V.Y. Part I: Impact of field-induced quantum confinement on subthreshold swing behavior of line TFETs. IEEE Trans. Electron. Devices 2013, 60, 4057-4064. [CrossRef]

14. Padilla, J.L.; Gamiz, F.; Godoy, A. A simple approach to quantum confinement in tunneling field-effect transistors. IEEE Electron. Device Lett. 2012, 33, 1342-1344. [CrossRef]

15. Padilla, J.L.; Alper, C.; Gamiz, F.; Ionescu, A.M. Assessment of field-induced quantum confinement in heterogate germanium electron-hole bilayer tunnel-field transistor. Appl. Phys. Lett. 2014, 105, 082108. [CrossRef]

16. Lim, S.K. Bringing 3D ICs to Aerospace: Needs for Design Tools and Methodologies. J. Inf. Commun. Converg. Eng. 2017, 15, 117-122. [CrossRef]

(C) 2018 by the authors. Licensee MDPI, Basel, Switzerland. This article is an open access article distributed under the terms and conditions of the Creative Commons Attribution (CC BY) license (http://creativecommons.org/licenses/by/4.0/). 